\title{
Metabolic associated fatty liver disease and cancer risk: causal role or epiphenomenon?
}

\author{
Ali Bayoumi ${ }^{1}$, Mohammed Shatat ${ }^{2}$, Mohammed Eslam $^{1}$ \\ ${ }^{1}$ Storr Liver Centre, Westmead Institute for Medical Research, Westmead Hospital and University of Sydney, Westmead, NSW, Australia; \\ ${ }^{2}$ Department of Internal Medicine, Faculty of Medicine, Minya University Hospital, Minya, Egypt \\ Correspondence to: Mohammed Eslam. Storr Liver Centre, Westmead Institute for Medical Research, Westmead Hospital and University of Sydney, \\ Westmead 2145, NSW, Australia. Email: mohammed.eslam@sydney.edu.au. \\ Comment on: Allen AM, Hicks SB, Mara KC, et al. The risk of incident extrahepatic cancers is higher in non-alcoholic fatty liver disease than obesity \\ - A longitudinal cohort study. J Hepatol 2019;71:1229-36.
}

Submitted Feb 14, 2020. Accepted for publication Feb 24, 2020.

doi: $10.21037 / \mathrm{hbsn} .2020 .03 .05$

View this article at: http://dx.doi.org/10.21037/hbsn.2020.03.05

The majority of global deaths are attributed to one of the noncommunicable diseases (NCDs), and cancer is projected to be the leading cause of death worldwide in the $21^{\text {st }}$ century (1). There were 18.1 million incident cancer cases worldwide in 2018, 43.8 million are living with cancer and 9.6 million deaths from cancer. One in 6 deaths is due to cancer and these numbers are projected to rise to 13 million by 2030 , more than that from tuberculosis, malaria and HIV/AIDS combined. In addition, the total annual global cost of cancer was estimated at around US\$1.16 trillion, representing a significant burden on health care systems and families (1).

Notably, approximately a third of cancer cases preventable and are attributed to behavioural and dietary risks, and an additional third treatable if diagnosed early. In this regard, numerous epidemiological studies and metaanalyses provided unequivocal evidence to demonstrate an association between obesity, as defined by body mass index (BMI) and at least 12 types of cancer, including liver, colorectal, pancreatic, esophageal, breast, kidney and bladder (2). Imbalances in dietary intake, energy homeostasis, and inappropriate physical activity fuel to a large extent, this global epidemic of obesity. According to the World Health Organization (WHO), the prevalence of overweight and obesity has almost tripled since 1975 and in 2016, 1.9 billion adults globally were overweight, of whom 650 million had obesity (3). Hence, obesity is projected to be soon replacing smoking as the leading preventable cause of cancer.
Nevertheless, not all fats are the same; ectopic fat accumulation, particularly in the liver and skeletal muscle entails higher risk for the development of insulin resistance and cardiometabolic abnormalities and adverse events, compared to total body adiposity. Thus, it is currently recognised that BMI is only an imprecise indicator of obesity as it does not assess body fat composition or central obesity (4,5).

In parallel with epidemics of obesity, the prevalence of metabolic associated fatty liver disease (MAFLD) (formerly known as NAFLD) that encompasses a significant accumulation of fat in the liver, has significantly increased affecting up to a quarter of the world's adult population and is currently a leading cause of chronic liver disease worldwide (6-8). As MAFLD are fundamentally intertwined with obesity and metabolic syndrome, it is not surprising that the clinical burden and consequences of MAFLD are not limited only to the risk of liver-related complications but it extends also to increase the rate and severity of various extrahepatic complications, including multiple extrahepatic cancers (9). Indeed, the leading cause of death in patients with MAFLD is cardiovascular disease, followed by extrahepatic malignancies and then liver-related complications (10). Thus, dissecting the effects of obesity and its associated metabolic consequences, from those mediated by MAFLD on extrahepatic cancers is important.

A recent study by Allen $e t$ al. (11) provides new insights on the impact of MAFLD, demonstrating that liver fat is a pivotal mediator in the association between obesity and 
the development of hepatic and extrahepatic cancer. This longitudinal study included a community cohort of 4,722 NAFLD and 14,441 age- and sex-matched control subjects followed up to 21 years with a median follow-up of 8 years. During this period, there were 2,224 incident cancers. The presence of MAFLD was associated with $90 \%$ greater risk of developing cancers after adjustment for potential confounders. The highest risk increase was demonstrated in liver cancer [incidence rate ratio (IRR) 2.8; 95\% CI, 1.65.1], followed by those for uterine, stomach, pancreas, then colon cancer. Interestingly, MAFLD was associated with an approximately two-fold increase in the overall risk of incident cancers in comparison to non-obese controls (IRR 2.0; 95\% CI, 1.5-2.9), while obesity had much less impact on cancer risk (IRR 1.2; 95\% CI, 0.9-1.6). Furthermore, this study demonstrated that obesity per say, in the absence of MAFLD did not significantly increase the risk compared to non-obese controls (IRR 1.0; 95\% CI, 0.4-1.4).

The next frontier is to go from the epidemiological association to the mechanistic explanation. Though a direct causal link between MAFLD and extrahepatic cancers is yet to be clarified, several mechanisms have been postulated. MAFLD is intimately interconnected with hepatic and systemic insulin resistance, which in accompanying with high-glucose environment can lead to oncogenic alterations and the promotion of various pathways involved in cell proliferation, such as MAPK/ERK, PI3K/Akt, Ras, and c-MYC. In addition, the low-grade systemic inflammation associated with MAFLD can induce mitochondrial dysfunction, release pro-carcinogenic cytokines and hormones and promote reactive oxygen species (ROS) accumulation. These events can lead to the activation of oncogenic pathways. Moreover, metabolic perturbation as occurs in MAFLD can induces wide epigenetic oncogenic changes and DNA mutations (12).

However, it remains challenging to ascertain causality and disentangle if liver fat itself, the intertwined endocrine activity of adipose tissue, or additional factors, such as microbiota changes and poor dietary intake are causative, given the complex and dynamic interaction between these factors. The possibility that MAFLD represents a robust surrogate of visceral obesity and thus has a stronger association with extrahepatic cancers than obesity, as assessed by BMI, also cannot be excluded.

An important question from this report is whether we can leverage the new knowledge to improve the management of patients and broaden opportunities for cancer control? Obviously, if this study is independently confirmed by others, it could help in risk stratification of patients and identification of those with increased risk of obesity-promoted malignancy, ultimately enabling more personalised surveillance approaches. Such knowledge would also help determine the optimal type of and time point for treatment and prevention strategies for obesity and MAFLD.

In conclusion, MAFLD likely plays a more critical role rather than obesity in the development of hepatic and extrahepatic cancers. Current measures of obesity seem to be imperfect and thus practical everyday tools to characterize excess adiposity are required. Though external validation of the findings is required, they are intriguing and are an impetus to undertake further research in order to address the questions raised.

\section{Acknowledgments}

Funding: None.

\section{Footnote}

Provenance and Peer Review: This article was commissioned by the editorial office of Hepatobiliary Surgery and Nutrition. The article did not undergo external peer review.

Conflicts of Interest: All authors have completed the ICMJE uniform disclosure form (available at http://dx.doi. org/10.21037/hbsn.2020.03.05). The authors have no conflicts of interest to declare.

Ethical Statement: The authors are accountable for all aspects of the work in ensuring that questions related to the accuracy or integrity of any part of the work are appropriately investigated and resolved.

Open Access Statement: This is an Open Access article distributed in accordance with the Creative Commons Attribution-NonCommercial-NoDerivs 4.0 International License (CC BY-NC-ND 4.0), which permits the noncommercial replication and distribution of the article with the strict proviso that no changes or edits are made and the original work is properly cited (including links to both the formal publication through the relevant DOI and the license). 
See: https://creativecommons.org/licenses/by-nc-nd/4.0/.

\section{References}

1. Bray F, Ferlay J, Soerjomataram I, et al. Global cancer statistics 2018: GLOBOCAN estimates of incidence and mortality worldwide for 36 cancers in 185 countries. CA Cancer J Clin 2018;68:394-424.

2. Lauby-Secretan B, Scoccianti C, Loomis D, et al. Body fatness and cancer-viewpoint of the IARC Working Group. New Engl J Med 2016;375:794-8.

3. Organization WH. Obesity and overweight. 2020. Available online: https://www.who.int/news-room/factsheets/detail/obesity-and-overweight

4. Eslam M, George J. Genetic contributions to NAFLD: leveraging shared genetics to uncover systems biology. Nat Rev Gastroenterol Hepatol 2020;17:40-52.

5. Eslam M, George J. Refining the role of epicardial adipose tissue in non-alcoholic fatty liver disease. Hepatol Int 2019;13:662-4.

6. Eslam M, Sanyal AJ, George J. MAFLD: A consensusdriven proposed nomenclature for metabolic associated fatty

Cite this article as: Bayoumi A, Shatat M, Eslam M. Metabolic associated fatty liver disease and cancer risk: causal role or epiphenomenon? Hepatobiliary Surg Nutr 2020;9(6):774-776. doi: $10.21037 / \mathrm{hbsn} .2020 .03 .05$ liver disease. Gastroenterology 2020;158:1999-2014.e1.

7. Younossi Z, Anstee QM, Marietti M, et al. Global burden of NAFLD and NASH: trends, predictions, risk factors and prevention. Nat Rev Gastroenterol Hepatol 2018;15:11-20.

8. Sarin SK, Kumar M, Eslam M, et al. Liver diseases in the Asia-Pacific region: a Lancet Gastroenterology \& Hepatology Commission. Lancet Gastroenterol Hepatol 2020;5:167-228.

9. Byrne CD, Targher G. NAFLD: a multisystem disease. J Hepatol 2015;62:S47-64.

10. Adams LA, Lymp JF, St Sauver J, et al. The natural history of nonalcoholic fatty liver disease: A population-based cohort study. Gastroenterology 2005;129:113-21.

11. Allen AM, Hicks SB, Mara KC, et al. The risk of incident extrahepatic cancers is higher in non-alcoholic fatty liver disease than obesity - A longitudinal cohort study. J Hepatol 2019;71:1229-36.

12. Stone TW, McPherson M, Darlington LG. Obesity and Cancer: Existing and New Hypotheses for a Causal Connection. Ebiomedicine 2018;30:14-28. 